\title{
Non-Monotonic-Offers Bargaining Protocol
}

\author{
Pinata Winoto \\ Dept. of Computer Science \\ University of Saskatchewan \\ Saskatoon, SK S7N5A9, Canada \\ piw410@mail.usask.ca
}

\author{
Gordon McCalla \\ Dept. of Computer Science \\ University of Saskatchewan \\ Saskatoon, SK S7N5A9, Canada \\ mccalla@cs.usask.ca
}

\author{
Julita Vassileva \\ Dept. of Computer Science \\ University of Saskatchewan \\ Saskatoon, SK S7N5A9, Canada \\ jiv@cs.usask.ca
}

\begin{abstract}
This paper discusses the strengths and weaknesses of non-monotonic-offers in alternating-offer bargaining protocol. It is commonly assumed that bargainers submit monotonic offers over time, which correspond to their belief revisions. However, through formal analysis and simulations, we are able to show that nonmonotonic-offers protocols can generate higher average surplus and lower breakdown rate compared to monotonic-offers protocols.
\end{abstract}

\section{Introduction}

The area of bargaining mechanisms has been well explored both in multi-agent systems and economics [1-12]. One of the properties, which are commonly agreed in the analyses of alternating-offers bargaining, is the (weak) monotonic (counter-) offers by bargainers, i.e. buyers/sellers may only insist on their previous offers or raise/reduce their offers monotonically until an agreement is reached. For instance, time dependent tactics [1, 2], behavior dependent tactics [1], and market-driven strategy [7], all have this monotonic-offers property. In [11], the authors show that the monotonic-offers property in the bargaining between two rational and self-interested agents is the consequence of the belief revision mechanism of those agents. Intuitively, if a buyer asks for $\$ 10$ to buy an item, but the seller insists on $\$ 20$, then the buyer may reduce its belief that the seller is willing to sell for $\$ 10$, which forces the buyer to revise its offer to a higher price rather than a lower one. However, this intuition is correct if the buyer believes that the seller's valuation (reservation price) will not change until the end of the bargaining. In this paper we will show that imposing non-monotonicoffers protocol (N-protocol, for short) in bargaining may be better than imposing monotonic-offers protocol (Mprotocol). Let us first look at an illustrative example below.

Scenario 1. Suppose that a buyer wants to buy a service (e.g. predicting the future price of stocks), which it needs most in a specific time (e.g., before the stock market opens). However, if it cannot get the service during that period, its utility from the service decreases over time, and becomes zero after a time deadline (e.g., after the market closes). Thus, the buyer's valuation of the service will be high when the market opens, and will decrease until the market closes (as shown as a downward line in Figure 1). Suppose that seller's valuation is constant over time (the horizontal line in Figure 1). Since both parties hold their information (valuations) privately, the initial spread (the difference between initial offers by buyer and seller) will be relatively big. The spread will decrease as the bargaining progresses (by sending offers and counter-offers). However, under M-protocol, the bargaining may be stuck even if both parties repeat the bargaining for several times,
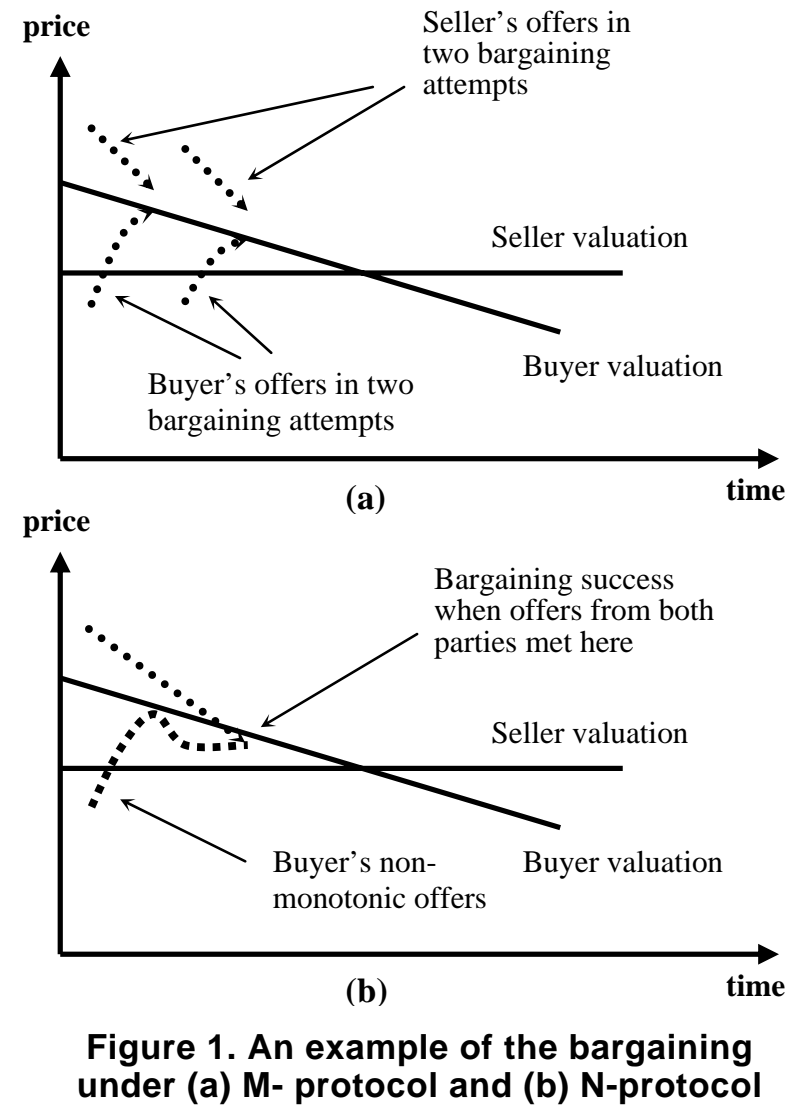
as illustrated in Figure 1(a). The failures are caused by the buyer who cannot resume the negotiation after its offer approaches its valuation, because its next offer will be higher than its next valuation. However, under N-protocol, the failure can be remedied as illustrated in Figure 1(b).

The illustration above only shows a partial scenario, which favors N-protocol. Indeed, some bargainers' decision process under $\mathrm{N}$-protocol may suppress the convergence of spread in reaching a concession, which will be explained later.

The paper is organized as follows. In the next section, we will show a formal analysis of $\mathrm{N}$-protocol when all agents are rational. Then, in section 3 we will provide simulation results using bounded rational agents and introducing some irrational agents whose behaviors cannot be analyzed formally. In section 4 , we will discuss the effect of our results and related work. Finally, we point out potential directions for future work in section 5 .

\section{Formal analysis}

Let us assume that all agents are rational, selfinterested and utility maximizers. For simplicity reasons, we will only focus on the buyer's perspective, which can be applied symmetrically for the seller.

\subsection{Basic model}

Let two agents bargain over a single-attribute item using alternating-offers protocol, in which the buyer shows a decreasing valuation over time, until time deadline, $\boldsymbol{T}_{\boldsymbol{d}}$ and the seller's valuation is unknown for the buyer. For simplicity, let each period (labeled by $t=1,2,3$, etc.) consist of a pair of decisions by the buyer and the seller where the buyer moves first. Also, suppose the bargaining variable is the item's price only, and both parties do not know any information about their opponents (e.g. time deadline, valuation, or bargaining strategy). Nonetheless, a buyer believes that offering a higher price may have a higher chance of being accepted by the seller, as stated in the following proposition.

Proposition 1. The buyer's belief $p\left(x_{t}\right)$ that its offer, $x_{t}$ is going to be accepted by a seller, is an increasing function of $\boldsymbol{x}_{t}$, if $\boldsymbol{p}\left(\boldsymbol{x}_{\boldsymbol{t}}\right)$ is independent of its preceding offers $\boldsymbol{x}_{1}, \boldsymbol{x}_{2}$, $\ldots, x_{t-1}$.

As a rational agent, a buyer will never offer a value that generates negative profit/surplus and it always prefers an offer that generates higher expected gain $E G$, which is defined as

$\mathrm{E} G_{t} \equiv\left(1-q_{t}\right)\left[p\left(x_{t}\right)\left(B_{t}-x_{t}\right)+\gamma\left(1-p\left(x_{t}\right)\right) \mathrm{E} G_{t+1}^{\prime}\right]+q_{t} B_{\phi}\left(1^{\prime}\right)$ where $\boldsymbol{q}_{t}$ is the likelihood of failure (breakdown) caused by the seller at time $\boldsymbol{t} ; \boldsymbol{B}_{\boldsymbol{t}}$ is the buyer valuation at time $\boldsymbol{t} ; \boldsymbol{\gamma}$ is the weight put for the expected gain in the next period; $E G^{\prime}{ }_{t+1}$ is the prediction of the expected gain made in the next period; and $\boldsymbol{B}_{\phi}$ is the buyer valuation if the negotiation breaks down. If the negotiation does not break down, which may happen by probability $\left(\mathbf{1}-\boldsymbol{q}_{t}\right)$, then two possible states may happen. The first state is that the bargaining succeeds immediately (by probability $p\left(x_{t}\right)$ ), which gives the buyer positive surplus $\left(\boldsymbol{B}_{t}-\boldsymbol{x}_{t}\right)$, denoted by $\operatorname{Sur}\left(\boldsymbol{x}_{\boldsymbol{t}}\right)$. The second state is that the bargaining proceeds to the next period, which may give the buyer expected gain $E G_{t+1}{ }_{t+1}$ in the future. Let us assume that no surplus is generated from a breakdown, thus equation ( 1 ') becomes

$$
\mathrm{E} G_{t}=\left(1-q_{t}\right)\left[p\left(x_{t}\right)\left(B_{t}-x_{t}\right)+\gamma\left(1-p\left(x_{t}\right)\right) \mathrm{E} G_{t+1}^{\prime}\right]
$$

Intuitively, as time goes by, the likelihood of breakdown increases, because both parties approach their time deadlines, or $\boldsymbol{q}_{\boldsymbol{t}+1}>\boldsymbol{q}_{\boldsymbol{t}}$. Assuming that $\boldsymbol{p}\left(\boldsymbol{x}_{\boldsymbol{t}}\right)$ does not change over time, and since $\boldsymbol{B}_{t+1}<\boldsymbol{B}_{t}$, then the buyer may expect that $\mathrm{E} \boldsymbol{G}_{t+1}^{\prime}<\mathrm{E} \boldsymbol{G}_{\boldsymbol{t}}$. It is important here to differentiate between $E \boldsymbol{G}^{\prime}{ }_{t+1}$ and the true value $\mathrm{E} \boldsymbol{G}_{\boldsymbol{t}+\boldsymbol{l}}$, because the latter may only be known at time $t+1$, i.e. after the buyer revises its belief. We may also assume that $\gamma<1$, and that the buyer will reduce $\boldsymbol{p}\left(\boldsymbol{x}_{\boldsymbol{t}}\right)$ if its offer, $\boldsymbol{x}_{\boldsymbol{t}}$ has not been accepted by the seller, or increase $\boldsymbol{p}\left(\boldsymbol{x}_{\boldsymbol{t}}\right)$ if the seller's counteroffer, $\boldsymbol{y}_{\boldsymbol{t}}$ is very close to $\boldsymbol{x}_{\boldsymbol{t}}$. Figure 2 shows an example of the buyer's belief at periods $\boldsymbol{t}$ and $\boldsymbol{t} \boldsymbol{n} \boldsymbol{n}$, i.e., after the seller drops its offer from $\$ 200$ to $\$ 190$.

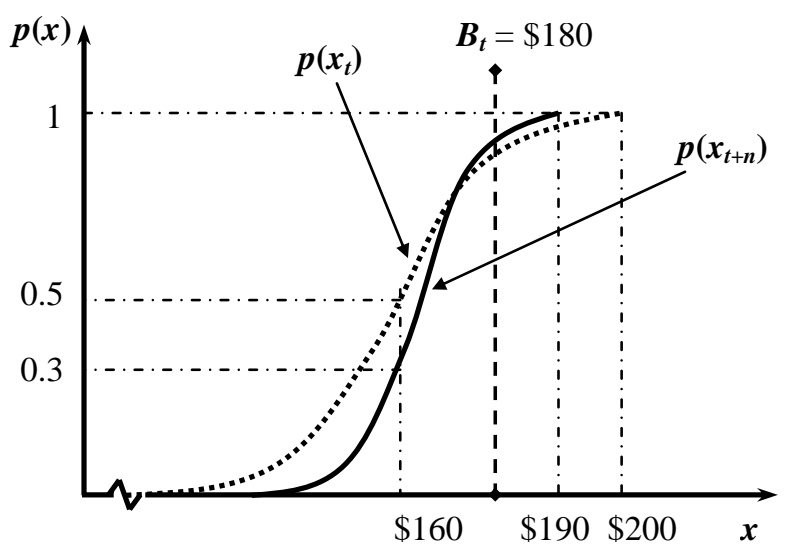

\section{Figure 2. An example of the shifting of a buyer's belief towards seller's acceptance rate}

Let us assume that the buyer's valuation is $\$ 180$ and its previous offer $\boldsymbol{x}_{\boldsymbol{t}}=\$ 160$, with $\boldsymbol{p}(\$ 160)=0.5$. If the seller reduces its offer from $\$ 200$ to $\$ 190$ after insisting for $\boldsymbol{n}$ periods, then the buyer may change its belief from $p\left(x_{t}\right)$ to $p\left(x_{t+n}\right)$. The new beliefs are higher for prices above $\$ 175$, and lower for prices below $\$ 175$ (the intersection of $\boldsymbol{p}\left(\boldsymbol{x}_{\boldsymbol{t}}\right)$ and $\left.p\left(x_{t+n}\right)\right)$. Thus, the buyer now believes that the chance of the seller accepting an offer of $\$ 160$ is 0.3 , which may force the buyer to raise its offer.

Given the definition of expected gain in equation (1), we can derive the next proposition regarding agent preference over an additional offer to a series of consecutive offers that ends up with a breakdown. 
Proposition 2. A series of consecutive offers $\left\langle x_{1}, x_{2}, \ldots\right.$, $\left.\boldsymbol{x}_{\boldsymbol{v}} \phi\right\rangle$ is preferred than $\left\langle\boldsymbol{x}_{\boldsymbol{1}}, \boldsymbol{x}_{2}, \ldots, \boldsymbol{x}_{\boldsymbol{t}-\boldsymbol{l}}, \boldsymbol{\phi}\right\rangle$ for $0 \leq \boldsymbol{x}_{\boldsymbol{t}}\left\langle\boldsymbol{B}_{\boldsymbol{t}}\right.$.

Proposition 2 states that an additional opportunity to submit an offer is always preferred than ending up with a breakdown, no matter what the value of additional offer is, i.e. $\boldsymbol{x}_{\boldsymbol{t}}$ could be higher, lower or equal to $\boldsymbol{x}_{\boldsymbol{t}-\mathrm{I}}$. The proof of this proposition is trivial because $\mathrm{E} \boldsymbol{G}_{\boldsymbol{t}}>0=\mathrm{E} \boldsymbol{G}_{\boldsymbol{\phi}}$.

As shown in Figure 1(a), a buyer's offer may be stuck at its valuation if its opponent does not accept it, which results in a breakdown. Thus, under M-protocol, there is always a risk of early breakdown if $\boldsymbol{x}_{t}>\boldsymbol{B}_{T d}$ (valuation at time deadline). Let us assume that there are two types of buyers, risk-seeking buyers, whose offer(s) $\boldsymbol{x}_{\boldsymbol{t}}>\boldsymbol{B}_{T d}$, and risk-averse buyers, whose offers $\boldsymbol{x}_{\boldsymbol{t}} \leq \boldsymbol{B}_{T \boldsymbol{T}}$. If we allow agents to repeat the bargaining after a breakdown as long as it is conducted before a time deadline $\boldsymbol{T}_{\boldsymbol{d}}$, and there is no cost of doing it, then it can easily be shown that riskseeking buyers outperform risk-averse buyers in terms of the success rate (probability of making a concession). However, if the repetition is restricted, e.g., due to a time delay on establishing a new bargaining session after a breakdown, which is a more realistic situation, then riskaverse buyers may outperform risk-seeking buyers in terms of the success rate. Intuitively, if the time delay needed on establishing a new bargaining session is relatively high when compared to $\boldsymbol{T}_{\boldsymbol{d}}$, then a risk-seeking agent (who maximizes expected gain) may be trapped in a lower success rate. However, this situation can be avoided in $\mathrm{N}$-protocol, which is equivalent to a frictionless and repeatable bargaining protocol (without any cost or delay).

In the next sections, further analysis that considers the sellers' acceptance criteria will be provided. Two types of acceptance criteria (evaluation functions) are considered.

\subsection{Type-I agent}

A type-I agent uses a typical evaluation function in accepting an offer by its opponent, i.e., accept an offer if it is at least as good as the counteroffer that would be sent by the agent in the next period. For instance, if a buyer plans to offer $\$ 5$ in the next period, while the seller is currently asking for $\$ 4$, then the buyer will accept the seller's current offer instead of offering $\$ 5$ in the next period. The evaluation criteria of type-I agents can be formulated in the following definition.

Definition 1. A type-I agent uses the following evaluation function in making its decision:

$\boldsymbol{I}_{t}= \begin{cases}\text { Withdraw } & \text { if } \boldsymbol{t}>\boldsymbol{T}_{\boldsymbol{d}} \text { or } \operatorname{Sur}\left(\boldsymbol{x}_{t+1}\right)<0 \text { for all available } \boldsymbol{x}_{t+1} \\ \text { Accept } & \text { if } \operatorname{Sur}\left(\boldsymbol{y}_{t}\right) \geq \operatorname{Sur}\left(\boldsymbol{x}_{t+1}\right) \geq 0 \\ \text { Counter offer if } \operatorname{Sur}\left(\boldsymbol{y}_{t}\right)<\operatorname{Sur}\left(\boldsymbol{x}_{t+1}\right)\end{cases}$

Where $\boldsymbol{I}_{\boldsymbol{t}}$ is the agent's decision at time $\boldsymbol{t} ; \boldsymbol{y}_{\boldsymbol{t}}$ is the offer by the agent's opponent at time $\boldsymbol{t}$, and $\boldsymbol{x}_{\boldsymbol{t}}+1$ is the agent's offer that will be proposed at time $\boldsymbol{t}+1$. The evaluation function above is also used in automated negotiation literature [1,
2]. Recently, Sim and Wang [8] have adopted fuzzy rules to modify the acceptance criteria of type-I agents. Under their criterion, an agent will accept an offer even though it is slightly worse than its next counteroffer. Nevertheless, if all bargaining agents are type-I agents, then buyers in $\mathrm{N}$ protocol will monotonically change their offers until they get close to their valuation in order to maximize their expected gain. The following proposition formalizes the above statement.

Proposition 3. Let all agents under N-protocol be type-I agents and let this be common knowledge. In order to maximize its expected gain, a buyer will monotonically increase its offers when $\boldsymbol{B}_{\boldsymbol{t}}-\boldsymbol{x}_{\boldsymbol{t}} \gg \boldsymbol{\gamma} \boldsymbol{\boldsymbol { E }} \boldsymbol{G}_{\boldsymbol{t}+1}$, and decrease its offers when $\boldsymbol{B}_{\boldsymbol{t}}-\boldsymbol{x}_{\boldsymbol{t}} \rightarrow \boldsymbol{\gamma} \mathrm{E} \boldsymbol{G}_{t+1}^{\prime}$.

Proof. (sketch) Since all agents are type-I and it is common knowledge, then all agents know that their offers will be accepted only if they are at least as good as their opponents' offers. From equation (1), we can derive the expected gain of a buyer in offering two different prices, $\boldsymbol{x}_{t}{ }^{1}$ and $\boldsymbol{x}_{t}^{2}$, as follow,

$$
\begin{aligned}
& \mathrm{E} G_{t}^{1}=\left(1-q_{t}\right)\left[p\left(x_{t}{ }_{t}\right)\left(B_{t}-x_{t}{ }_{t}\right)+\gamma\left(1-p\left(x_{t}{ }_{t}\right)\right) \mathrm{E} G^{1}{ }^{1}{ }_{t+1}\right] \\
& \mathrm{E} G_{t}^{2}=\left(1-q_{t}\right)\left[p\left(x^{2}{ }_{t}\right)\left(B_{t}-x^{2}{ }_{t}\right)+\gamma\left(1-p\left(x^{2}\right)\right) \mathrm{E} G^{2}{ }_{t+1}\right]
\end{aligned}
$$

Let $\boldsymbol{x}_{t}{ }^{1}=\boldsymbol{x}_{t}^{2}+\Delta \boldsymbol{x}$, then from proposition 1, we have $\boldsymbol{p}\left(\boldsymbol{x}_{t}{ }^{1}\right)$ $>p\left(x_{t}^{2}\right)$. Since the buyer can offer any price at time $t+1$, which maximizes its expected gain, then $\mathrm{E} \boldsymbol{G}{ }^{1}{ }_{t+1} \approx \mathrm{E} G^{2}{ }{ }_{t+1}$. Moreover, since a rational buyer will choose a higher offer only if $E G_{t}{ }_{t}>E G_{t}{ }_{t}$, then we only need to compare the part inside brackets, i.e., $p\left(x_{t}^{I}\right)\left(B_{t}-x_{t}^{I}\right)+\gamma\left(\mathbf{1}-p\left(x_{t}^{I}\right)\right) \mathrm{E} G^{I}{ }^{1}{ }_{t+1}$ $>p\left(x_{t}{ }_{t}\right)\left(B_{t}-x^{2}{ }_{t}\right)+\gamma\left(1-p\left(x_{t}^{2}\right)\right) \mathrm{E} G^{2}{ }_{t+1}$. By replacing $p\left(x^{2}\right)$ with $p\left(x_{t}{ }_{t}\right)-\Delta p$, and $x_{t}^{2}$ with $x_{t}^{I}-\Delta x$, then we can derive the precondition for $\mathrm{E} G_{t}{ }_{t}>E G_{t}{ }_{t}$ as,

$$
\frac{\Delta p}{\Delta x}>\frac{p\left(x_{t}^{2}\right)}{B_{t}-x^{1}{ }_{t}-\gamma \mathrm{E}{G^{1}}^{1}{ }_{t+1}}
$$

From the precondition above, we can conclude that $\boldsymbol{x}_{t}{ }_{t}>$ $\boldsymbol{x}_{t}^{2}$ will be chosen if $\boldsymbol{B}_{t}-\boldsymbol{x}_{t}{ }_{t} \gg \gamma \mathrm{E} \boldsymbol{G}_{t+1}{ }_{t+1}$. As the offer increases, then the slope $\Delta p / \Delta x$ decreases (see Figure 2) and $\boldsymbol{B}_{t}-\boldsymbol{x}_{t}{ }_{t}$ also decreases; therefore, the buyer has less incentive to increase its offer near its valuation.

Proposition 3 describes the self-adjustment of the buyer's offer in order to maximize its expected gain. A buyer will aggressively increase its offers at the beginning of bargaining until they are closer to its valuation, and then the buyer may adjust its offer by lowering it (nonmonotonic offer) or stay on it. The convergence of the buyer's offers is guaranteed as stated in proposition 4 below.

Proposition 4. Under N-protocol, if all agents are type-I agents and this is common knowledge, then $\boldsymbol{x}_{\infty} \rightarrow \boldsymbol{B}_{\infty}$.

Proof. (sketch) Since the likelihood of breakdown increases over time, i.e. $\boldsymbol{q}_{\infty} \rightarrow 1$, and $E G_{t+1}=\left(1-\boldsymbol{q}_{t+1}\right)$ [ 
$\left.p\left(x_{t+1}\right)\left(B_{t+1}-x_{t+1}\right)+\gamma\left(1-p\left(x_{t+1}\right)\right) E G_{t+2}\right]$; thus, $\mathrm{E} G_{\infty} \rightarrow 0$. Moreover, if $\boldsymbol{x}_{t}{ }_{t}$ has not been accepted for a certain time, then the buyer's belief that all offers lower than or equal to $\boldsymbol{x}_{\boldsymbol{t}}$ will be accepted also decreases to 0 . Therefore, the RHS of equation (2) converges to zero, which forces the buyer to increase its offer to be higher than $\boldsymbol{x}_{\boldsymbol{t}}$. And if this process continues, then $\boldsymbol{x}_{\infty} \rightarrow \boldsymbol{B}_{\infty}$.

From propositions 3 and 4 , we can derive several properties of type-I buyers as follows:

- If the pressure from the likelihood of breakdown increases, then the buyer's offer will converge quickly to its valuation;

- If the buyer's valuation decreases sharply, then its offer may also decrease (due to the decreasing value of the denominator of RHS in equation (2));

- If the buyer's belief function has a steep curve near its valuation, then its offers will also converge quickly to its valuation.

From our previous analysis, we do not concern failure into our analysis. However, in the real situation, users may be concerned about the failure more than about the surplus. In such model, agents are required to find concession as soon as possible, even with zero surplus. Thus, without being concerned the amount of surplus, the best strategy for a buyer is to offer its valuation over time. Intuitively, agents in $\mathrm{N}$-protocol will outperform agents in $\mathrm{M}$ protocol, as stated in proposition 5 below.

Proposition 5. If type-I agents are only concerned about the success rate, then $\mathrm{N}$-protocol is preferred over $\mathrm{M}$ protocol.

Combining Proposition 2 through 5, we can show that $\mathrm{N}$-protocol is better than M-protocol for type-I buyers.

Theorem 1. N-protocol is at least as good as M-protocol for type-I agents.

Proof. (sketch) Firstly, agents in N-protocol can always choose offers that maximize their expected gain (by Proposition 3), while agents in M-protocol can only do this for increasing offers. Therefore, the expected gain made by agents in $\mathrm{N}$-protocol is greater than that in $\mathrm{M}$ protocol. This is true not only for a decreasing valuation, but also for an increasing one, because it is possible that $\boldsymbol{B}_{t+1}-\gamma E \boldsymbol{G}_{t+2}^{\prime}>\boldsymbol{B}_{t}-\gamma \mathrm{E} \boldsymbol{G}_{t+1}^{\prime}$ (cf. equation (2)), or the buyer has incentive to decrease its offer at time $t+1$ to maximize its expected gain. Secondly, buyers in Mprotocol may get stuck in their valuation (in case of decreasing valuation), which incurs some cost in order to repeat the bargaining. In contrast, buyers under N-protocol will never get stuck, and by Proposition 2, this is preferred. While this is not applied in the case of increasing valuation, allowing N-protocol causes no cost. Finally, for agents who are only concerned with success rate, Nprotocol provides higher opportunity than M-protocol (Proposition 5)

\subsection{Type-II agent}

In addition to the evaluation criterion used by type-I agent, a type-II agent uses an additional evaluation function in accepting an offer by its opponent, accept an offer if it is perceived to generate an optimal surplus. For instance, if the buyer's current valuation and next offer are $\$ 100$ and $\$ 80$ respectively, while the seller's current offer is $\$ 90$, then the buyer will accept the seller's current offer if it predicts that the seller will increase the price instead of reducing it in the next period. The rationale is that some agents may be myopic and risk-averse towards breakdown in the future. While they also behave as type-I agents, this additional evaluation criterion makes type-II agents more 'vulnerable' to a manipulative strategy in N-protocol. For instance, a seller may manipulate its offer frequently by increasing and decreasing it in attempt to mislead a buyer to accept it. Definition 2 below formalizes the type-II agents evaluation criterion.

Definition 2. A type-II agent uses the following evaluation function in making its decision:

$\boldsymbol{I}_{t}= \begin{cases}\text { Withdraw } & \text { if } \boldsymbol{t}>\boldsymbol{T}_{d} \text { or } \operatorname{Sur}\left(\boldsymbol{x}_{t+1}\right)<0 \text { for all available } \boldsymbol{x}_{t+1} \\ \text { Accept } & \text { if } \operatorname{Sur}\left(\boldsymbol{y}_{t}\right) \geq \operatorname{Sur}\left(\boldsymbol{x}_{t+1}\right) \text { or } 0 \leq \operatorname{Sur}^{e}\left(\boldsymbol{y}_{t+1}\right) \leq \operatorname{Sur}\left(\boldsymbol{y}_{t}\right) \\ \text { Counter offer if } \operatorname{Sur}\left(\boldsymbol{y}_{t}\right)<\operatorname{Sur}\left(\boldsymbol{x}_{t+1}\right)\end{cases}$ Where $\operatorname{Sur}^{e}\left(y_{t+1}\right)$ is the predicted value of $\operatorname{Sur}\left(y_{t+1}\right)$.

Under this criterion, the agents' expected gain is still the same as with type I agents as the offer increases, but slightly different as the offer decreases. A price reduction may increase the probability of acceptance, because it may be a signal of a local optimum for the seller. Therefore, the analysis of the increasing price is the same as that in the proof of proposition 3, but the analysis of the decreasing price is slightly different. Let the expected gain of $\boldsymbol{x}_{t}$ is

$$
\mathrm{E} G_{t}=\left(1-q_{t}\right)\left[p\left(x_{t}\right)\left(B_{t}-x_{t}\right)+\gamma\left(1-p\left(x_{t}\right)\right) \mathrm{E} G_{t+1}^{\prime}\right]
$$

Then a reduction of the price to $\boldsymbol{x}_{\boldsymbol{t}}{ }^{\prime}<\boldsymbol{x}_{t-1}$, will yield

$$
\begin{array}{r}
\mathrm{E} G_{t}=\left(1-q_{t}\right)\left[\left(p\left(x_{t}^{\prime}\right)+p\left(x_{t}^{\prime}\right)^{\prime}\right)\left(B_{t}-x^{\prime}{ }_{t}\right)+\right. \\
\left.\gamma\left(1-p\left(x_{t}^{\prime}\right)-p\left(x_{t}^{\prime}\right)^{\prime}\right) \mathrm{E} G_{t+1}^{\prime}\right]
\end{array}
$$

Where $\boldsymbol{p}\left(\boldsymbol{x}_{t}\right)^{\prime}$ ' is the belief of the acceptability of current offer $\boldsymbol{x}_{t}{ }_{t}$ when it is not better than the seller's next offer (from seller's perspective). Thus, $\boldsymbol{p}\left(\boldsymbol{x}_{t}^{\prime}\right)$ ' depends on $\boldsymbol{x}_{\boldsymbol{t}}$ and the ratio of sellers who perceived it as the signal of local optimal. Using similar analysis in the proof of Proposition 3, we can derive that a buyer will reduce its offer under the following condition:

$$
\frac{\Delta p}{\Delta x}<\frac{p\left(x_{t}^{\prime}\right)}{\left(B_{t}-x_{t}-\gamma \mathrm{E} G_{t+1}^{\prime}\right)}+\frac{p\left(x_{t}^{\prime}\right)^{\prime}}{\left(x_{t}-x_{t}^{\prime}\right)} \frac{\left(B_{t}-x_{t}^{\prime}-\gamma \mathrm{E} G_{t+1}^{\prime}\right)}{\left(B_{t}-x_{t}-\gamma \mathrm{E} G_{t+1}^{\prime}\right)}
$$

The second term of the RHS in equation (4), which is always a positive value, is an additional feature that incorporates $\boldsymbol{p}\left(\boldsymbol{x}_{t}{ }_{t}\right)$. Similar to the situation in type-I agents, we may conclude that that buyers may benefit form $\mathrm{N}$ protocol, because they can maximize their expected gain either by increasing or decreasing their offers. It can also be shown that their offers will eventually approach their 
valuations. And if type-II agents are only concerned about the success rate, then $\mathrm{N}$-protocol is better than M-protocol. The following propositions formalize the above statement.

Proposition 6. Under N-protocol, if all agents are type-II agents and it is common knowledge, then $\boldsymbol{x}_{\infty} \rightarrow \boldsymbol{B}_{\infty}$.

Proof. (Sketch). The proof is similar to the proof of proposition 4 , i.e. $E \boldsymbol{G}_{\infty} \rightarrow 0$ and $\boldsymbol{p}\left(\boldsymbol{x}_{\boldsymbol{t}}\right) \rightarrow 0$ if $\boldsymbol{x}_{\boldsymbol{t}}$, has not been accepted for a certain time. In addition to it, we also need to prove that $\boldsymbol{p}\left(\boldsymbol{x}_{t}{ }_{t}{ }^{\boldsymbol{\prime}} \rightarrow 0\right.$ if $\boldsymbol{x}_{\boldsymbol{t}}{ }$ has not been accepted for a certain time. This is true, because if $\boldsymbol{x}_{t}{ }_{t}$ has not been accepted for a certain time, then either the seller did not perceive a price reduction as the signal of optimal offer or $\boldsymbol{x}_{\boldsymbol{t}}$ is too low to generate surplus for seller. Thus, the RHS of equation (4) converges to zero, which forces the buyer to increase its offer higher than $\boldsymbol{x}_{\boldsymbol{t}}$. Thus, $\boldsymbol{x}_{\infty} \rightarrow \boldsymbol{B}_{\infty}$.

Proposition 7. If type-II agents are only concerned about the success rate, then $\mathrm{N}$-protocol is preferred than $\mathrm{M}$ protocol.

The proof is similar to the proof of Proposition 5.

Theorem 2. N-protocol is at least as good as M-protocol for type-II agents.

The proof is similar to the proof of Theorem 1.

According to Theorems 1 and 2, we conclude that $\mathrm{N}$ protocol is better than M-protocol in terms of success rate and expected gain for buyers. However, this conclusion based on formal analysis can be objected since it relies on the strict assumption of agents' rationality and being utility maximizers, which is not realistic in the real world. For example, what is the best belief revision for the buyers in our analysis above? Can a buyer really maximize its expected gain according to equations (2) or (4)? Similar objection has been addressed widely to a lot of research in the areas of distributed AI and economics. If the formal analysis method is thus limited, then the only alternative is to show the N-protocol superiority through experimentation with artificial and/or human agents. In the next section, we will use a simulation to test the $\mathrm{N}$-protocol from the seller's perspective, in order to prove the validity of our formal analysis for sellers in more relaxed conditions, where agents are myopic and have bounded rationality.

\section{Simulation}

\subsection{Experimental design}

First, 100 pairs of upward valuation (both buyers and sellers have non-increasing valuations over time) are generated randomly under a pre-specified range. Two experiment parts are designed, based on the protocol and the strategies used by agents:

- $\quad$ Agents use random strategies in bargaining (part 1)
- Agents use reactive (behavior-dependent) strategies in bargaining (part 2) [1]

The part 1 experiment can be divided into four groups according to the strategies used by sellers:

- Risk-averse seller (R-averse): a seller who offers monotonic price and will not offer any price below the maximum valuation (in this case $\$ 100$ );

- $\quad$ Risk-seeking seller (R-seeking): a seller who offers monotonic price but may offer any price above its valuation;

- Nonmonotonic-offer seller (N-seller): a seller who offers any price above its valuation and may increase it as its valuation increases, but only when it is stuck on its valuation;

- Nonmonotonic with random change (NR-seller): a seller who is similar to $\mathrm{N}$-seller, except that it may increase its price randomly (with probability equals to 0.1 ) in order to attract type-II buyers.

We subdivide each group of the part 1 experiments into four treatments based on the strategies used by sellers and buyers shown in Table 1. Thus, a total of 16 groups of experiments are conducted in part 1 , and each group is repeated 300 times for each pair of valuations, resulting in 480000 trials. In the part 1 experiment, agents only follow random strategies, i.e. sellers (buyers) randomly increase (decrease) their offers until the valuations are reached. Depending on the agents' characteristics, some may raise/drop their offers faster than others, but they never take their opponents' offers/behavior into consideration. The realization of a type-I agent is by using the following method,

- if my opponent's current offer generates higher positive surplus than my offer which will be sent in the next period, then accept my opponent's current offer

The realization of a type-II agent is by adding the following method in addition,

- if my opponent's current offer generates higher positive surplus than the previous one, do nothing

- if my opponent's current offer generate lower positive surplus than the previous one, accept it

Table 1. Four treatments based on agents' evaluation

\begin{tabular}{|c|c|c|c|}
\hline & \multicolumn{2}{|c|}{ Seller } \\
\hline & & Type-I & Type-II \\
\hline \multirow{2}{*}{ Buyer } & Type-I & S1B1 & S2B1 \\
\hline & Type-II & S1B2 & S2B2 \\
\hline
\end{tabular}

The experiments in part 2 are almost the same as in part 1, except that most agents consider their opponents' offers, and react accordingly. Four reactive strategies are considered, which are slightly different from those used in $[1,7]$ :

- Tit-for-tat: the proponent's move is the same as the opponent's previous move 
- Tit-for-2tat: the proponent's move is the same as the opponent's previous two moves

- Tat-for-tit: the proponent's move is the reverse of the opponent's previous move

- Spread-driven: the proponent's move tries to reduce the spread of negotiation by a constant fraction

Each experiment in part 2 consists of agents with the four reactive strategies above plus agents with random strategies. Eight different treatments are conducted in part 2: the four different treatments given in Table 1, and two different protocols for each of them (N-protocol and Mprotocol). For the statistical analysis, each possible combination will be repeated more than 30 times. Totally, we have conducted 720000 trials in part 2. Since two reactive agents may stand on their offers (e.g. two tit-for-tat agents will always use the same strategies if met), we use a "tiebreaker" mechanism -- an agent will not stand on its current offer for more than n-periods. Moreover, we assume a very high cost in repeating a bargaining session. Thus, if an agent is stuck in its valuation, then we will consider it as a breakdown. Table 2 shows the general parameters used in both part 1 and part 2 of our experiments.

Table 2. Parameters used in the experiment

\begin{tabular}{|l|c|}
\hline \multicolumn{1}{|c|}{ Parameters } & Values \\
\hline Maximum bargaining periods & 99 \\
\hline Sellers and buyers' initial valuation & $\$ 50-\$ 85$ \\
\hline Sellers and buyers' final valuation & $\$ 100$ \\
\hline Increment of valuation & $\$ 5$ \\
\hline Range of sellers' initial offers & $\$ 100-\$ 120$ \\
\hline Range of buyers' initial offers & $\$ 30-\$ 50$ \\
\hline Min. increment/decrement of offers & $\$ 1$ \\
\hline Max. increment/decrement of offer & $\$ 5$ \\
\hline
\end{tabular}

3.1.1. Agents' valuations. Figure 3 shows four representative pairs of agents' valuations, which are generated randomly. The vertical axis represents the price, and the horizontal axis represents the time line (in periods). The thick line represents the buyer's valuation in each period, and the thin line represents the seller's valuation. The transaction may be made within the area where the thick line is above the thin line, or when both of them are on the same horizontal line. Near the end of the bargaining period, both lines always overlap at $\$ 100$. This gives us a higher assurance of a success if the bargaining extends to the deadline, which may favor risk-averse sellers in part 1 or our experiment.

3.1.2. Evaluation criteria. In both part 1 and part 2, two main variables are recorded for evaluation purposes:

- total surplus generated from each group experiment, in terms of the sum of surplus for both buyers and sellers;

- number of breakdown/success.
Based on these two values, three types of information are retrieved: average surplus, average surplus per success transaction, and success rate.
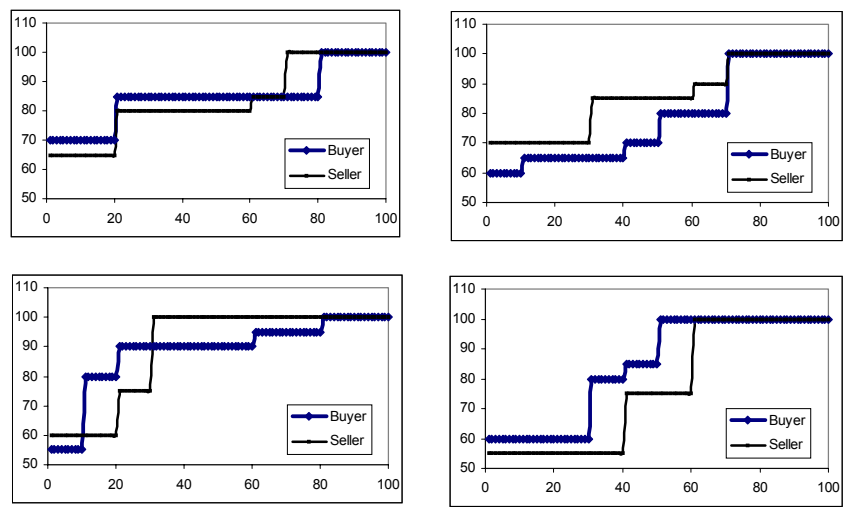

Figure 3. Examples of four pairs (out of 100) of upward valuation used in the experiments

\subsection{Results}

Table 3 shows that agents in N-protocol (the two bottom rows) generate higher surplus compared to agents in M-protocol (the two upper rows). The success rates of Nprotocol are also higher compared to the setting where sellers are risk-taking in M-protocol $\left(2^{\text {nd }}\right.$ row $)$. These results justify our theoretical analysis that $\mathrm{N}$-protocol favors negotiation under sellers' upward valuations.

Table 3. Result of experiment in part 1

\begin{tabular}{|l|r|r|r|}
\hline & \multicolumn{1}{|c|}{$\begin{array}{c}\text { Ave. sur- } \\
\text { plus }\end{array}$} & $\begin{array}{c}\text { Ave. surp./ } \\
\text { transaction }\end{array}$ & Success rate \\
\hline R-averse & 6.657 & 6.967 & 94.903 \\
\hline R-seeking & 7.098 & 8.773 & 80.399 \\
\hline N-seller & 10.053 & 10.272 & 97.901 \\
\hline NR-seller & 10.109 & 10.820 & 93.323 \\
\hline
\end{tabular}

If we compare the results of each group of strategies used by buyers and sellers (as shown in Table 1), we find out that the effect of the strategy used by sellers and buyers is not significant in N-protocol (Figure 4). Smaller average surpluses are only observed in the case when the sellers are of type-II and the buyers are of type-I (shown as S2B1). In contrast, in M-protocol, much smaller average surpluses are observed when the sellers are of type-I (see Figure 5, in case S1B1 and S1B2). The result suggests that type-II sellers outperform type-I sellers in Mprotocol.

Introducing NR-sellers in N-protocol reduces the success rate, as predicted. The effort to increase the price in order to convince the buyer to make concessions earlier will prolong the bargaining, thus increasing the risk of 
breakdown. However, as expected, it generates higher total surplus, since more concessions are made and surplus is generated for both parties.

In part 2, the success rate and the average surplus generated are higher in $\mathrm{N}$-protocol, as shown in Table 4. $100 \%$ success rate is gained under our experiment in $\mathrm{N}$ protocol, which much higher than $65 \%$ in M-protocol. All the results shown are statistically significant under t-test for confidence level $>95 \%$. This result justifies that $\mathrm{N}$ protocol is better than M-protocol in terms of average surplus gained by bargainers and their success rate, when both parties have increasing valuations.

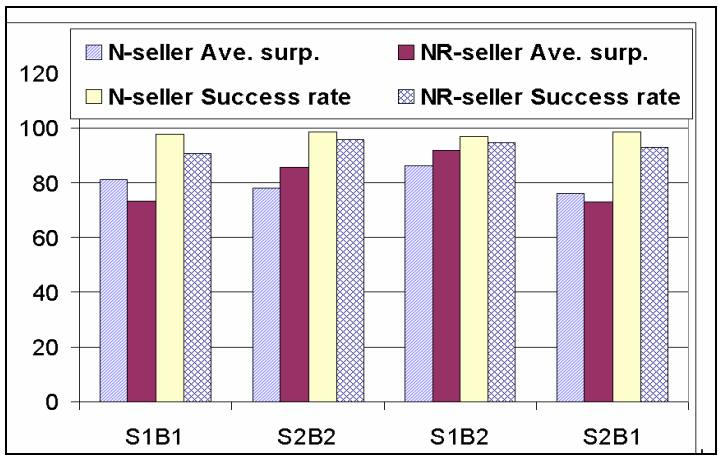

Figure 4. A comparison between $\mathrm{N}$-seller and NR-seller in $\mathbf{N}$-protocol (average surplus is normalized)

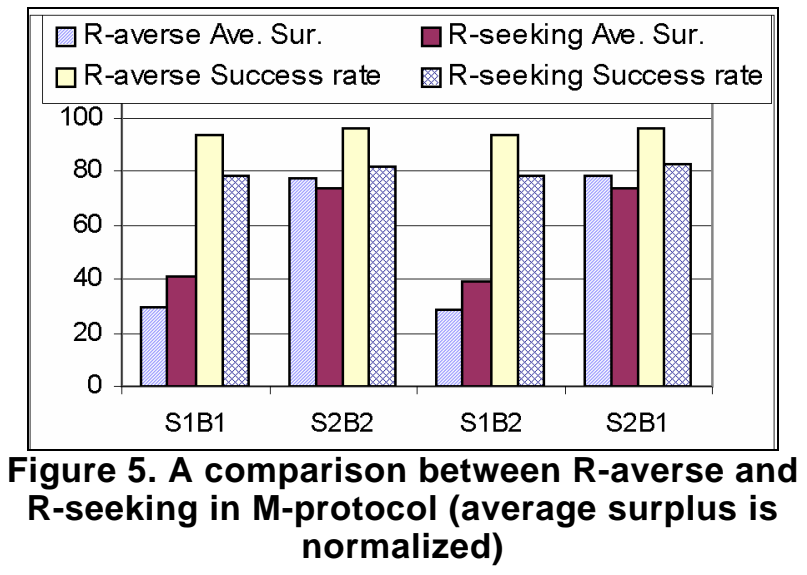

Table 4. Result of experiment in part 2

\begin{tabular}{|c|c|c|}
\hline & Average surplus & Success rate \\
\hline M-protocol & 8.37 & 65 \\
\hline N-protocol & 9.62 & 100 \\
\hline
\end{tabular}

\section{Discussion}

As mentioned earlier in this paper, an alternative solution for an agent who has diminishing valuation includes re-opening negotiation with the same or a new opponent every time a weak deadline has been met. However, this situation is not simulated in our experiment here because it is less realistic. Intuitively, if two bargainers have met before, then they may restart their initial offers closer than if they do not know each other in the first place, which may speed up the convergence to the concession. However, there are several weaknesses of this mechanism:

1. If the opponent is programmed to restart the bargaining session using its initial offer, then the chance of breakdown increases, because of the diminishing valuation of the proponent.

2. If there are other party(-ies) queuing for the bargaining, then a termination within two agents may reduce the chance for both to encounter each other again, which increases the chance of both to start their bargaining by offering their initial offer (to other new parties).

3. There may exist costs to restart the bargaining, as indicated previously.

In addition, if two bargainers can repeat their bargaining frictionless in an M-protocol, then the efficiency (in term of expected gain and success rate) gained by them is at most as high as the efficiency gained in N-protocol, which becomes the upper bound of the efficiency of $\mathrm{M}$ protocol.

Finally, in N-protocol, agents who use a simple bargaining strategy, such as random strategy or tit-for-tat, can gain higher efficiency (in term of success rate and surplus) compared to agents in M-protocol. This suggests that Nprotocol may work better than M-protocol for most types of agents, including those able to maximize expected gain, as shown in the theoretical analysis. However, this may not be true if we have the following agents in the bargaining:

- Irrational agents, who increase or decrease their offers arbitrarily; this can be avoided by the restriction of M-protocol.

- Misinformed agents, who cannot accept a nonmonotonic offer, therefore retreat from the bargaining immediately. These agents may perceive a nonmonotonic offer as a sign of a lack of seriousness of their opponent, or as a sign of a prolonged bargaining, or as a sign of a higher likelihood of breakdown. Thus, they will retreat from the bargaining, and try to find a new opponent.

- Nasty agents, who use non-monotonic offers to threaten their opponents, delay the bargaining, or mislead their opponents' beliefs. This can be accomplished if agents have less pressure from time deadline or breakdown.

More issues may arise by allowing N-protocol, but as shown in both the theoretical analysis and the simulation, $\mathrm{N}$-protocol is better than M-protocol under certain conditions.

\subsection{Related work}


Many works in bargaining have addressed several issues such as the deadline of the bargaining [5], the strategies under incomplete information [1,7], agents with limited resources [4], and learning by buyer and seller [12]. Most studies of bargaining simplify the model by imposing several assumptions, such as common knowledge of deadline, valuation, risk-neutral attitude, etc. [2, 4, 5, 6]. Even if the exact value is unknown, many works assume that the probability of it is known (incomplete information). Thus, most of the bargaining problems are solvable using game-theoretic analysis, at the cost of a less applicable domain [3]. In competitive negotiation bargainers will withhold their private information, since revealing private information will subject them to exploitation by their opponents. Consequently, it leads to a more complex model, which is not solvable using game theory. The alternative solution is to use heuristic approach in the design of negotiating agents $[1,7,8,9,10,12]$. Our work here aims to the latter case, where agents are blind to their opponents, and can only observe the offer/counter offer by its opponent. However, adopting $\mathrm{N}$-protocol may also alter the result of some analysis in game theory. For instance, let both the buyer and the seller know that the buyer's deadline is earlier than the seller's, and both valuations are decreasing over time and privately known. Then the best strategy for the seller in M-protocol is to wait until the buyer's deadline and accept the buyer's offer as long as it generates positive surplus. This constitutes the sub-game perfect equilibrium, because there is no incentive for both seller and buyer to offer before the buyer's deadline, and there is no incentive for the seller to accept any offer before the deadline. However, under N-protocol, the buyer may offer a higher price at the beginning of negotiation and reduce it along its valuation. Under this strategy, the best response of seller is to accept the price that generates highest surplus, which depends on the slope of seller's valuation. Thus, the equilibrium points in M-protocol and $\mathrm{N}$-protocol will be different.

\section{Future work}

For our future work, we aim to extend our experiment by using more sophisticated agent strategies, such as incorporating a learning mechanism to predict the opponent's valuation, as in [10]. Since the current simulation is conducted for the case of increasing valuation for both buyer and seller, other cases, such as decreasing valuation for buyer and increasing valuation for seller, or both with decreasing valuations but with different slope and deadline need to be studied. Finally, we also plan to extend the simulation by introducing various nasty/irrational agents.

\section{Acknowledgement}

This research has been supported by the Canadian Natural Sciences and Engineering Research Council (NSERC).

\section{References}

[1] Faratin, P., Sierra, C. and Jennings, N. R. Negotiation Decision Functions for Autonomous Agents. Int. J. of Robotics and Autonomous Systems, 24(3-4), 1998, 159-182.

[2] Fatima, S. S., Wooldridge, M. J. and Jennings, N. R. Optimal Negotiation Strategies for Agents with Incomplete Information. In ATAL-01, Seattle, Washington, 53-68.

[3] Jennings, N. R., Faratin, P., Lomuscio, A. R., Parson, S., Sierra, C., Wooldridge, M. Automated Negotiation: Prospects, Methods, and Challenges. Journal of Group Decision and Negotiation, 10 (2), 2001, 199215.

[4] Larson, K. and Sandholm, T. W. An Alternating Offers Bargaining Model for Computationally Limited Agents. In AAMAS-02, Bologna, Italy, 135-142.

[5] Kraus, S., Wilkenfeld, J. and Zlotkin, G. Multiagent Negotiation under Time Constraints. Artificial Intelligence, 75, 1995, 297-345.

[6] Rubinstein, A. A Bargaining Model with Incomplete Information about Time Preferences. Econometrica, 53, 1985, 1151-1172.

[7] Sim, K. M. A Market-driven Model for Designing Negotiation Agents. Computational Intelligence, 18(4), 2002, 618-637.

[8] Sim, K. M. and Wang, S. Y. Designing Flexible Negotiation Agent with Relaxed Decision Rules. In IAT03, Halifax, Canada, 282-289.

[9] Somefun, K., Gerding, E., Bohte, S. and Poutré, H. Automated Negotiation and Bundling of Information Goods". In AMEC $V$, Melbourne, Australia, July, 2003

[10] Vassileva, J. and Mudgal, C. Negotiation with Incomplete and Uncertain Information: Trading Help in a Distributed Peer Help Environment. In Parsons S., Gmytrasiewicz P., Wooldridge M. (eds.) GameTheoretic and Decision-Theoretic Agents, Kluwer Academic Publishers, 2002, pp. 337-354.

[11] Winoto, P., McCalla, G. and Vassileva, J. An Extended Alternating-Offers Bargaining Protocol for Automated Negotiation in Multi-agent Systems. In CoopIS 2002, Irvine, CA, 179-194.

[12] Zeng, D. and Sycara, K. Bayesian learning in negotiation. International Journal of Human-Computer Studies, 48, 1998, 125-141. 